NIST Advanced Manufacturing Series 100-36

\title{
Uncertainties for Machine Tool \\ Modeling
}

Guodong Shao

William Stann

This publication is available free of charge from:

https://doi.org/10.6028/NIST.AMS.100-36

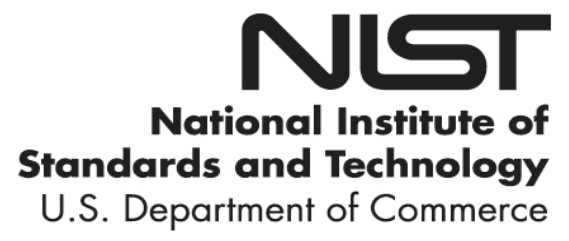




\title{
Uncertainties for Machine Tool Modeling
}

\author{
Guodong Shao \\ Systems Integration Division \\ Engineering Laboratory
}

William Stann

Loyola University

Baltimore, Maryland

This publication is available free of charge from:

https://doi.org/10.6028/NIST.AMS.100-36

September 2020

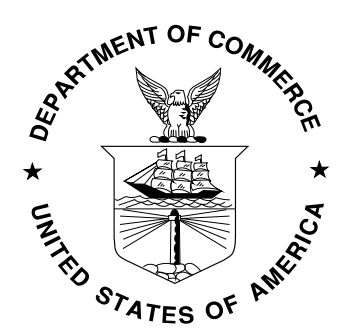

U.S. Department of Commerce

Wilbur L. Ross, Jr., Secretary

National Institute of Standards and Technology Walter Copan, NIST Director and Undersecretary of Commerce for Standards and Technology 
Certain commercial entities, equipment, or materials may be identified in this document in order to describe an experimental procedure or concept adequately. Such identification is not intended to imply recommendation or endorsement by the National Institute of Standards and Technology, nor is it intended to imply that the entities, materials, or equipment are necessarily the best available for the purpose.

National Institute of Standards and Technology Advanced Manufacturing Series 100-36

Natl. Inst. Stand. Technol. Adv. Man. Ser. 100-36, 20 pages (September 2020)

This publication is available free of charge from: https://doi.org/10.6028/NIST.AMS.100-36 


\begin{abstract}
Machine tool models play an important role in supporting decision-making for machine tool procurement, process planning, and production scheduling in manufacturing. Due to modeling uncertainties, however, it is challenging to create a machine model that accurately and dynamically represents the real machine. Modeling uncertainties and errors can be introduced during the model development process (i.e., when a machine model is created from scratch) and the model conversion process (i.e., when a machine model in one format needs to be converted into another format, e.g., from a vendorspecific format to a neutral format such as the STandard Exchange of Product Data (STEP)). This paper identifies these uncertainties and provides a methodology to help ensure correct conversion of the coordinate system from one definition to another. Examples are provided to illustrate the methodology. As a fundamental method, this methodology will help improve model accuracy by compensating multiple modeling errors. It also supports the synchronization between machine models with real machines when building digital twins of the machine tools by adjusting the coordinate system offsets continuously. Digital twins of machine tools would be an integrated solution for addressing most of the modeling uncertainties in order to constantly monitor the status of the machine tool, dynamically update the model parameters, and in turn optimally control the machine tool.
\end{abstract}

\title{
Keywords
}

Machine Tool Modeling; Digital Twin; Smart Manufacturing; Uncertainty; Standards. 


\section{Table of Contents}

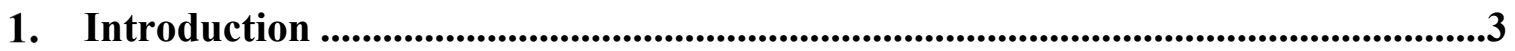

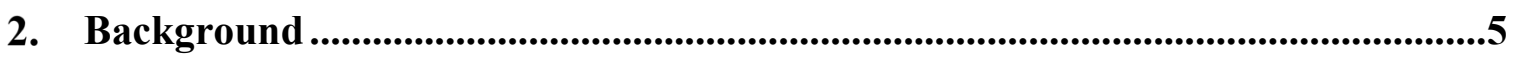

2.1. Machine Tool Modeling Scenarios ........................................................................

2.2. Machine Tool Model Conversion........................................................................6

3. Uncertainties for Machine Tool Modeling .......................................................6

4. Methodology for Converting Coordinate Systems ..............................................9

4.1. Coordinate System Definitions .................................................................

4.2. Coordinate System Conversion between Different Systems ..............................10

5. Digital Twins of Machine Tools...................................................................................14

6. Conclusion and Future Work....................................................................................15

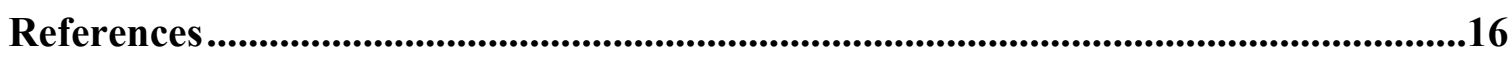

\section{List of Figures}

Fig. 1. Mapping matrix for coordinate system conversion from GCS to Link Frame ..... 10

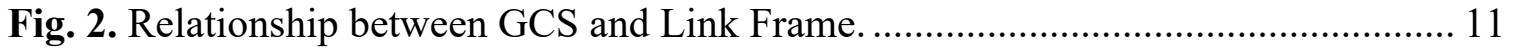

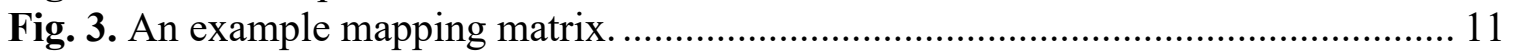

Fig. 4. Matrix expression for points.......................................................................... 12

Fig. 5. Complete point conversion equation............................................................ 12

Fig. 6. An example of converting GCS to Link Frame in the context of a machine tool. 12

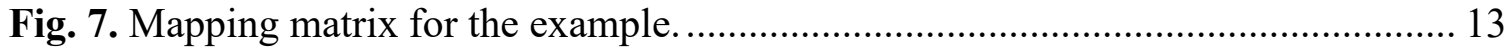

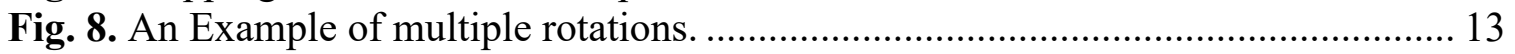

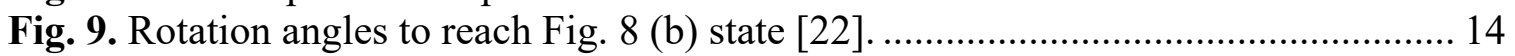

Fig. 10. Resulting mapping matrix of the rotation example...................................... 14 


\section{Introduction}

Smart Manufacturing Systems (SMS) are fully integrated, collaborative manufacturing systems that respond in real time to meet changing demands and conditions in the factory, in the supply network, and in customer needs [1].

Manufacturing companies are investing to digitize their processes and equipment to realize SMS, and digital modeling of machine tools is crucial to that effort.

As the most fundamental equipment and the most important resource in many manufacturing enterprises, a machine tool is used to produce parts through a subtractive cutting process. It contains four basic elements: (1) an energy source, (2) a means to keep the workpiece secure, (3) a means to keep cutting tools secure and orient the cutting tool, and (4) a means to control all the other means [2].

A machine tool model is a digital representation of the machine tool and its functionalities. Machine tool models describe the configuration of a machine, replicate the geometric shapes of the machine components, and specify the kinematic relationships between these machine components. The kinematics model of a machine tool describes the motion constraints for the machine components that relate to each other [3] and enables the simulation of the machining process. The machine model can be used by users for decision-making throughout the life cycle of the machine tool. For example, it can be used to support machine tool procurement by virtually and remotely evaluating machining capability before committing any investment, and it also supports process validation, production planning, and routing [4] [5] by helping identify manufacturing issues and correct the issues before production. Usually, a machine model is developed using a Computer-aided (CAx) tool, which may include Computer Aided Design (CAD), Computer Aided Manufacturing (CAM), or Computer Aided Engineering (CAE).

A CAx tool provides a virtual environment that enables the modeling and simulation of machining processes with a representation of the static and dynamic behavior of the machine tool [4]. However, because it is impossible to perfectly model all the behaviors and uncertainties of the machine tool, assumptions must be made during the machine tool model development process to simplify the model. For example, one assumption is that the machine body is rigid and does not experience compliance. Another assumption is that the machine body does not expand at higher operating temperatures.

To create an accurate machine tool model to realistically reflect the dynamic behavior of the physical machine, all the uncertainties must be considered. Multiple models with different purposes may need to be integrated together to achieve this goal. Eventually, digital twins of the machine tools may be needed to address these modeling uncertainties and errors. A digital twin of a machine tool is a digital representation of the machine tool and is data driven. The data need to be collected from the physical machine in real time using both internal and external sensors. The near real time data can be collected continuously (e.g., MTConnect stream data) or periodically (e.g., a daily measurement using meters). Depending on the application, a digital twin may cover both the modeling of the machine tool and the machining process. The digital twin model of a machine can be corrected, adjusted, or updated based on real-world measurements from the physical 
machine. In turn, the dynamically updated model helps make informed decisions. The integration between the digital twin and its physical twin is the biggest difference between digital twins and off-line models. As a modeling software, CAx tools will support digital twin implementations and SMS development. However, the use of CAx tools also brings challenges for model reuse and model integration.

There are a variety of commercial CAx tools from different vendors. These tools have been used by manufacturers to model their products and equipment to support design, operation, and maintenance activities.

As more machine tool models become available, both the benefits and the difficulty of model reuse and integration increase. Because each vendor has its own CAx environment with a specific format, even same-purpose tools from different vendors can cause issues for model sharing and exchanging. It is impractical for users to acquire and use all the tools, and the necessary learning curves would be considerable. As a result, redundant efforts are often required to re-create the same machine model using different CAx tools within a company; machine models with complex kinematics may be difficult or very time-consuming to remodel. This motivates manufacturers to seek solutions that support standard representations of their resources, products, and equipment, e.g., to represent machine models using a neutral format, which can be accepted and recognized by most vendor systems, if not all. Some vendors will refuse this idea because they would like to protect their own intellectual property (IP) of the kinematic models. Others will do it due to customers' demands.

Kinematics models from machine design provide error budgets, which control the accumulative errors in parts and assemblies. Therefore, kinematics simulation can provide error constraints for dynamic models that are used to predict the dynamic behavior of a machine tool. Representing kinematics models in a neutral format makes it easier to integrate it with these dynamic models of the machine tool.

Standards development efforts have been made to represent geometrical and functional information independent of any particular system and implementation method [5]. ISO 10303, the STandard Exchange of Product Data (STEP), has been implemented by most vendors and most CAx tools can import and export geometric models in this standard format. However, the definition of kinematics information in STEP has not been implemented by vendors and applied in practice and industry [6]. Extra efforts have to be made to develop interfaces to help convert vendor-specific kinematic models to the STEP standard representations [3] [7].

During the model conversion, there might also be issues affecting the accuracy and validity of the machine model. This paper identifies possible uncertainties arising from model creation and model conversion. It particularly focuses on the methodology of converting coordinate systems between different systems, provides examples for explaining the methodology, and discusses the ultimate solution, i.e., digital twins of machine tools. 
The rest of the paper is organized as follows: Section 2 provides background information. Section 3 identifies, in general, the uncertainties of machine tool modeling. Section 4 focuses on the methodology for coordinate system conversion during model conversation and model correction, with examples. Section 5 discusses digital twins of machine tools. Finally, Section 6 concludes the paper and discusses future work.

\section{Background}

Smart Manufacturing depends on modeling and analysis to produce decisions that lead to across-the-board operational improvements. Digital twins of machine tools or machine tool models can help ensure that production is ready to operate accurately the first time. There are many perspectives on models. A digital twin could contain many types of models such as machine capability models. cutting tool life model, quality control model, energy consumption models, processing time (scheduling) models, and thermal models for building Heating, Ventilation, and Air Conditioning (HVAC) design. A few machine modeling scenarios are listed below.

\subsection{Machine Tool Modeling Scenarios}

For equipment procurement, machine tool models can help identify the right machine to satisfy customers' specific needs. When planning to purchase a new machine tool, marketing material and machine specification are not enough for factory customers to be certain on whether or not the entire work envelop is "useable" since motion error varies across the work envelop. A model of the machine tool with kinematics will allow customers to simulate the machine's functionalities and better understand the machine's capability, capacity, safety, and ergonomics. It also enables easy virtual comparison with other candidate machines.

Machine tool models can help process planning by enabling analyses of the mechanical and functional properties such as the ability to perform different types of machining operations. Machine tool kinematics, tool change, and pallet change are modeled and described. Feed rates and spindle speeds can be calculated based on the combination of workpiece and tool material along with the type of operation being performed. A comparison needs to be made, though, between the required feeds and speed for the part and that available from the machine tool per axis. Axis velocity and acceleration can help machining time analysis. Static and dynamic behavior are also modeled for predicting manufacturing performance.

Machine tool models are also useful for factory design and layout planning. 3D machine tool models can be arranged into a realistic 3D factory model based on not only the functional relationships of the machines, but also dimensions, size, orientations of the machines. Because oftentimes there are some physical limitations and constraints in existing facilities for how to set up the new layout. Multiple layout options can be tried out and an optimal design can be derived using simulation. The dimensions and characteristics of the machine tool and its subsystems (control cabinet, process fluid handling, chip conveyors, power requirements, coolant system, and process fluid, etc.) 
described within the model are critical information when planning optimal factory layout. [8]. This information is provided by the vendor of the machine tool.

\subsection{Machine Tool Model Conversion}

STEP consists of a set of standards to facilitate data modeling throughout the entire lifecycle of a product [9]. The STEP information models can be categorized into application protocols (AP) or integrated resources (IR). APs are developed for specific application domains, e.g., AP 203 for aerospace, AP 214 for automotive, and AP 242 for integrating the kinematics, geometry, and assembly models. IRs are context-independent, e.g., ISO 10303-105 defines an IR for specifying the structure, motion, and analysis for kinematics mechanism [10].

Currently, only the geometry representation in STEP has been widely adopted by vendors and AP 242 is still work-in-progress and has not been implemented in vendors' CAx tools [11]. To convert a machine tool kinematics model developed using a commercial CAx tool to the STEP format, customized interfaces need to be developed. Li et al. [3] have made such an effort by developing a translator using Java-based Standard Data Access Interface (JSDAI) [12] for combining geometric data in the STEP format with kinematics data collected from a Siemens' NX machine model. Bärring et al. [7] have used a similar approach to develop a STEP generator for converting a Creo machine model to STEP AP 242 representation. During the model conversion, different coordinate system definitions in the source and the target systems need to be carefully handled and converted to ensure the validity of the machine model. Beside potential coordinate system conversion errors, there are many more uncertainties that impact the accuracy and thus the usefulness of the machine model.

\section{Uncertainties for Machine Tool Modeling}

Machine tool models are intended to help manufacturers shorten production lead time, improve product quality, and decrease manufacturing costs. However, there are challenges during the model design, building, and executing processes.

Uncertainties exist in machine tool modeling because of innate differences between the model and the physical machine tool the model represents. Most systematic uncertainties in machine tool modeling come from design simplifications. These simplifications are normally for those physical system behaviors that are too complex to model accurately. In these cases, assumptions have to be made, e.g., the assumption of a rigid machine body, which treats the machine frame as a non-bending component during modeling. These simplifications create variations and overall relative inaccuracies between virtual simulations and the actual machining processes. By identifying these uncertainties, considerations can be made during the modeling process to increase the accuracy and the credibility of the machine model.

These errors can be divided into two categories: random errors and systematic errors. While it is very difficult to find the sources of the random errors and predict when they 
will occur, systematic errors have root causes and can be measured and accurately predicted through modeling [13]. It is thus impossible to include random errors in machine models, but systematic uncertainties can be identified, analyzed, and addressed.

Machine models are inevitably simplified and idealized representations of axis limits and orientations, rather than true descriptions of the production machine whose components are manufactured and assembled within tolerances and are further subject to wear. Therefore, dynamic models must be updated over time to describe the current condition of the machine, the model only remains accurate for as long as the most recent measurement data truly represents the machine. There are a lot of critical "unmodelled effects" that impact the accuracy of the machine model. Longstaff et al. discussed these errors and the measurement issues that can have a negative influence on the accuracy of the model [14]:

1) Pseudo-static geometric errors include (a) Translational error $E_{m n}$, which is an error in the $\mathrm{m}$ axis direction caused by the motion of the $\mathrm{n}$ axis, e.g., Exx is a linear positioning error, while EXY is the straightness of the $\mathrm{Y}$ axis in the $\mathrm{X}$ axis direction; (b) Rotational error $\mathrm{E}_{\mathrm{kn}}$, which is an error of rotation about the $\mathrm{k}$ axis experienced as the $\mathrm{n}$-axis is moved. $\mathrm{k}$ is defined as $\mathrm{A}, \mathrm{B}, \mathrm{C}$ for rotation about the $\mathrm{X}, \mathrm{Y}$, and $\mathrm{Z}$ axes respectively, e.g., $E_{A X}$ is rotation of the $X$ axis about the $X$ axis (roll) and $E_{B Z}$ is rotation of the $\mathrm{Z}$ axis about the $\mathrm{Y}$ axis. Longstaff et al. highlighted [15] that the methods that model the errors are compromises between exactness, available computing power, and measurement technology. These errors can be modified by inertial forces due to shifting load, acceleration, poor support, etc.

2) Effect of build-up of tolerances: Tolerances are usually placed upon each of the individual error sources when specifying a machine for purchase, conducting maintenance or applying compensation.

3) Thermal errors: The thermal effect is often estimated as a linear multiplier of the temperature by the coefficient of thermal expansion of either the dominant or the scale material. This, however, is far too simplistic. Such an error not only affects machining, but also impacts the expected or calibrated performance of the machine. Therefore, temperature effects need to be carefully measured, analyzed, and modeled.

4) Non-rigid body errors: Although machine tools are generally designed to be stiff, the assumption of completely rigid machine bodies is inaccurate and introduces a modeling uncertainty. A good example of such error is cutting tool deflection, which means that the cutting tool will bend when the force of cutting overcomes the stiffness of the tool. Less effort has been focused on modeling the actual rigidity of machine cases than for other machine tool errors [16], because it is perceived as less significant when compared to geometric and thermal errors. However, this "finite stiffness" error can manifest itself as a self-induced distortion due to moving mass or as a response to the load of a moving workpiece, which can change during operation when material is removed.

5) Static and dynamic load-induced errors: Samir and Tunde discuss these types of errors [17], which are caused by forces such as the weight of the working material, the cutting forces generated during the machining, or the axis acceleration load 
resulting from the displacement of masses of the machine components [13]. These errors can create a deviation between the ideal geometry in models and the physical system in the form of angular deflection of the machine frame. This can be caused by vibration in the cutting fixture and cutting tool due to constructive simple harmonic motion, by linear displacement of the cutting tool's functional point generated by resistance experienced by the tool, or even by static loads such as gravitational forces on the machine frame [18]. These errors cannot be corrected with simple calibration as other common errors can be. Therefore, models that consider the errors are needed.

6) Dynamic errors: When measuring machines to calibrate static models, any dynamic data must be handled because the static and dynamic states of the machine are different. Dynamic effects of a machine tool include contouring errors, gain and scale mismatch, and vibration. The dynamics of the machine tool can be broadly split into the deviation from the rigid-body geometric assumption and the interpolation effects in translating the command movement into a path profile.

7) Translating errors: Vendor-specific machine models are defined geometrically and kinematically with respect to a default global cartesian coordinate system. This coordinate system is defined around a "Ground," which is an immovable part of a mechanical product in the global coordinate system. By standard practice, this is usually located in the bed of the machine [18]. Machine geometry errors caused by thermal deformation, static and dynamic loading, or the wear on components can affect the relative measurement and positioning of kinematic components between their intended placement in the model and their actual positioning with respect to the global coordinate system. In addition, the correctness of the model needs to be maintained when converting the model from a vendor-specific format to another format such as STEP. Geometry in STEP is defined by Link Frame, a form of a local coordinate system where each kinematic component has its own coordinate system and relative geometry. Each Link Frame has a coordinate system that is defined around a non-rigid origin, meaning that the coordinate system is free to move within the 3D space. In STEP, on the other hand, measurement such as positioning data and placement data is defined by mapping one Link Frame with respect to another [10]. If one kinematic link is misaligned or does not depict the exact position of the physical link due to any modeling error induced during the conversion process, any Link Frame defined with respect to that frame will also have an associated geometric, and eventually kinematic, error. STEP's geometry definition in Link Frame allows for greater error margins because spatial relationships between links are defined with respect to each other and not to a rigid, immobile, body.

There is not a single model or a tool that can address all the errors listed above. Multiple models with different focuses may need to be integrated together. Digital twins will help correct or update the machine model by using real-time measurement data as model inputs. In the next section, we focus on translating uncertainty and discuss a methodology for converting coordinate systems between different systems. 


\section{Methodology for Converting Coordinate Systems}

As discussed in the previous section, a coordinate system conversion could be a viable solution for compensation of static or dynamic induced errors that involve deflection and also help guarantee the correctness of the model when converting from one format to another. In this section, we first discuss the definitions of various coordinate systems and then provide a methodology that helps convert different coordinate systems, e.g., those defined in CAx tools and in STEP.

\subsection{Coordinate System Definitions}

Different coordinate systems in machine tools, CAx tools, and subsequent modeling include [19] [20]:

- The World Coordinate System (WCS): WCS is a coordinate system defined by the CAD system and around which all design geometry is constructed. The CAM system users cannot change the WCS but can define all its coordinate systems in relation to WCS if they want. Different CAM tools may have different default orientations of the WCS (e.g., the XY planes can serve as the top views in some tools, but as the front views in others.

- The Machine Coordinate System (MCS): the natural coordinate system of a machine tool defined by the machine tool manufacturer. It is the machine's internal origin point and known as "machine home." When the machine is powered up, it goes through a homing cycle and each axis is moved until it hits a limit switch. In some CAx tools, the MCS and WCS are coincident and aligned by default, in others, because of a default orientation issue, MCS must be adjusted to align with WCS. MCS is also known as absolute coordinate systems or Global Coordinate Systems (GCS).

- The Program Coordinate System (PCS): PCS is the coordinate system about which all toolpath or program points are computed and referenced. On the machine tool, PCS is equivalent to the workpiece coordinate system. PCS is defined or changed by the machine operator based on the job requirements to have a correct work offset. The machine tool controller interprets all program point coordinates with respect to PCS.

- Tool Coordinate System (TCS): references the tool (and tool tip) to its associated machine carriage and ultimately to the frame, table, and workpiece. It can also help describe the location of any metrology component attached in place of the tool during dimensional metrology. The origin may be at the gage line and spindle axis intersection. TCS helps set or adjust tool offsets.

- Model Coordinate System: the reference space of the model with respect to which all model geometrical data is stored. This is the only coordinate system that the software recognizes when storing or retrieving geometrical information in or from a model database. 
- Carriage Coordinate System: rigidly associated with each machine carriage to model the position and/or path of the tool.

- Frame Coordinate System: rigidly associated with the frame of the machine.

Once the coordinate systems have been defined with respect to the machine carriages etc., modeling and subsequent metrology can be used to find the position of the tool within MCS. Modeling can be performed by mathematically switching the reference coordinate systems in which the location of a point is known. This reference system switching is called a transformation or basis change. In this paper, we call it coordinate systems conversion.

\subsection{Coordinate System Conversion between Different Systems}

Commercial CAx applications such as Creo use GCS as their default coordinate system, in which all geometry and kinematics are defined. STEP uses a local coordinate system, which is also called "Link Frame" or the local coordinate system of a link [10]. Local coordinate systems in STEP are positioned with respect to each other and not to a single global coordinate system. Therefore, to convert a machine model developed using CAx tools to STEP, coordinate system conversion is required.

A process that enables efficient conversion of coordinate systems is called transformation or mapping. When converting, mapping maintains both coordinate system definitions, and merely changes how a point is described. Mapping as proposed and outlined by Zeid [21] includes three key components: (1) a homogeneous transformation matrix, (2) a coordinate (or series of coordinates) to be converted, and (3) matrix multiplication. Slocum provides a more detailed description of this topic [13].

The most complex aspect of mapping is the generation of the mapping matrix. For each coordinate system conversion, only one mapping matrix is required. The mapping matrix is composed of two parts, the rotation matrix $\mathrm{R}$ and the translation vector $\mathrm{T}$. The columns of the rotation matrix represent the unit vectors of the $\mathrm{X}, \mathrm{Y}$, and $\mathrm{Z}$ axes of the GCS coordinate system in the new local coordinate system, respectively. The translation vector represents the vector that points from the origin of the new local coordinate system to the GCS. The matrix form is shown in Fig. 1. The relationship between GCS and Link Frame is shown in Fig. 2.

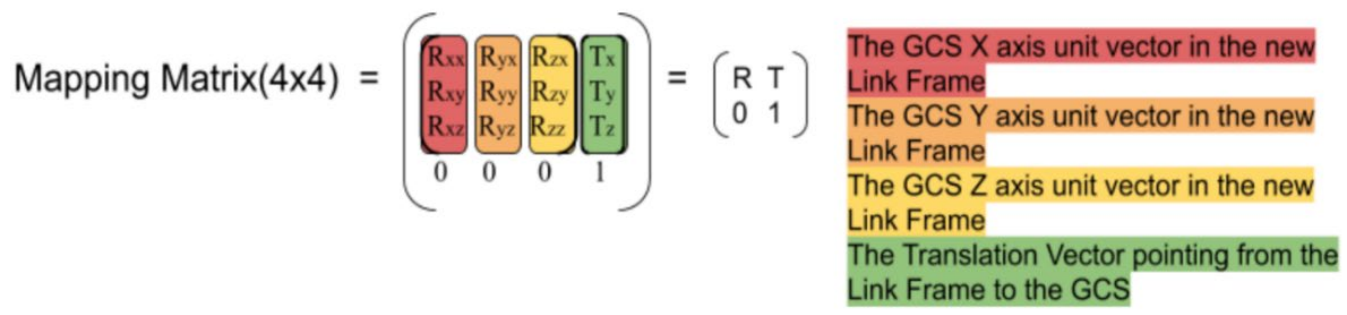

Fig. 1. Mapping matrix for coordinate system conversion from GCS to Link Frame 


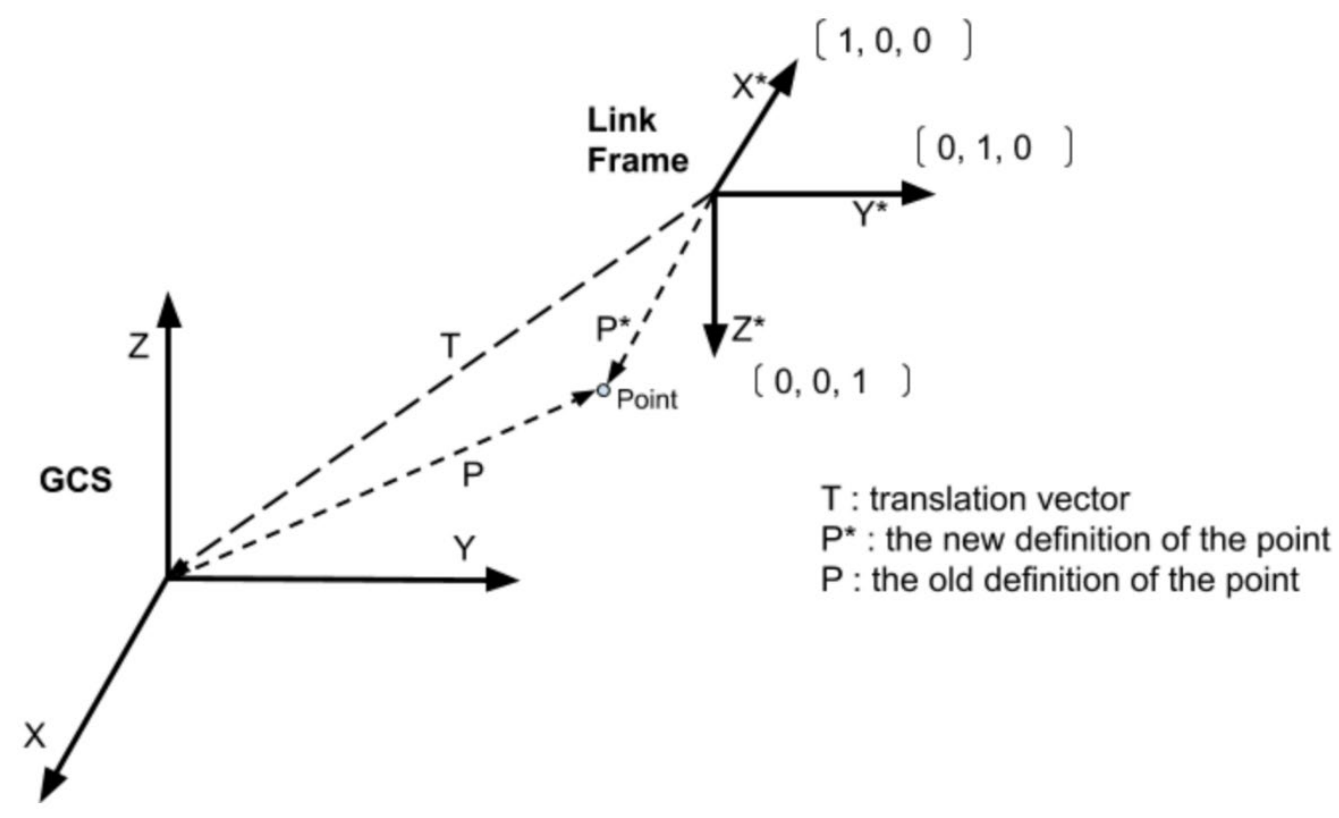

Fig. 2. Relationship between GCS and Link Frame.

For example, to find the new point $\left(\mathrm{P}^{*}\right)$ with respect to the Link Frame, we need to multiply (using matrix multiplication) the mapping matrix by the point $(\mathrm{P})$ that is defined with respect to the GCS.

If the transformation that converts the GCS to the Link Frame is a rotation of $180^{\circ}$ around the $\mathrm{Y}$ axis and a translation vector component of [Dx, Dy, Dz], the mapping matrix is shown in Fig. 3.

$$
\text { Given Mapping Matrix : }\left(\begin{array}{rrrr}
-1 & 0 & 0 & \mathbf{D x} \\
0 & 1 & 0 & \mathbf{D} y \\
0 & 0 & -1 & \mathbf{D z} \\
0 & 0 & 0 & 1
\end{array}\right)
$$

Fig. 3. An example mapping matrix.

More complicated rotation matrices may be required for rotations on multiple axes. Using the degrees of rotation about each axis and basic trigonometry, the vector components of each axis can be calculated and manually input into the mapping matrices if the CAx software does not provide the orientation and components automatically.

To comply with the rules of matrix multiplication, the coordinates of a point as described by GCS must be expressed in a (4x1) matrix. It will take the form of (X, Y, Z, 1) as shown in Fig. 4. The output in the new local coordinate system will be in the same form of matrix $\left(\mathrm{X}^{*}, \mathrm{Y}^{*}, \mathrm{Z}^{*}, 1\right)$. To have the correct $3 \mathrm{D}$ representation of the point, the fourth row (the 1) needs to be removed. 


$$
P=\left(\begin{array}{l}
X \\
Y \\
Z \\
1
\end{array}\right) \quad P^{*}=\left(\begin{array}{l}
X^{*} \\
Y^{*} \\
Z^{*} \\
1
\end{array}\right)
$$

Fig. 4. Matrix expression for points.

The final component of the conversion method is the formula that utilizes matrix multiplication (Fig. 5). Equation solvers can be easily developed using Java or C++.

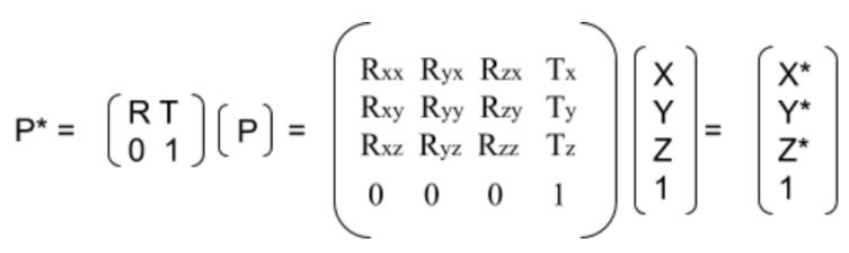

Fig. 5. Complete point conversion equation.

The example in Fig. 6 assumes that the GCS is positioned at the base of the machine tool. We would like to find the position of the coordinate point that defines the spindle tip with respect to the Link Frame of the spindle. The green arrow pointing from the Link Frame to the GCS is the translation vector. That vector can be derived by working backward from the position data of the origin of the Link Frame. The Link Frame coordinate system is rotated $90^{\circ}$ clockwise about the Z-axis relative to the GCS. Based on this information, the mapping matrix can be generated and is shown in Fig. 7.

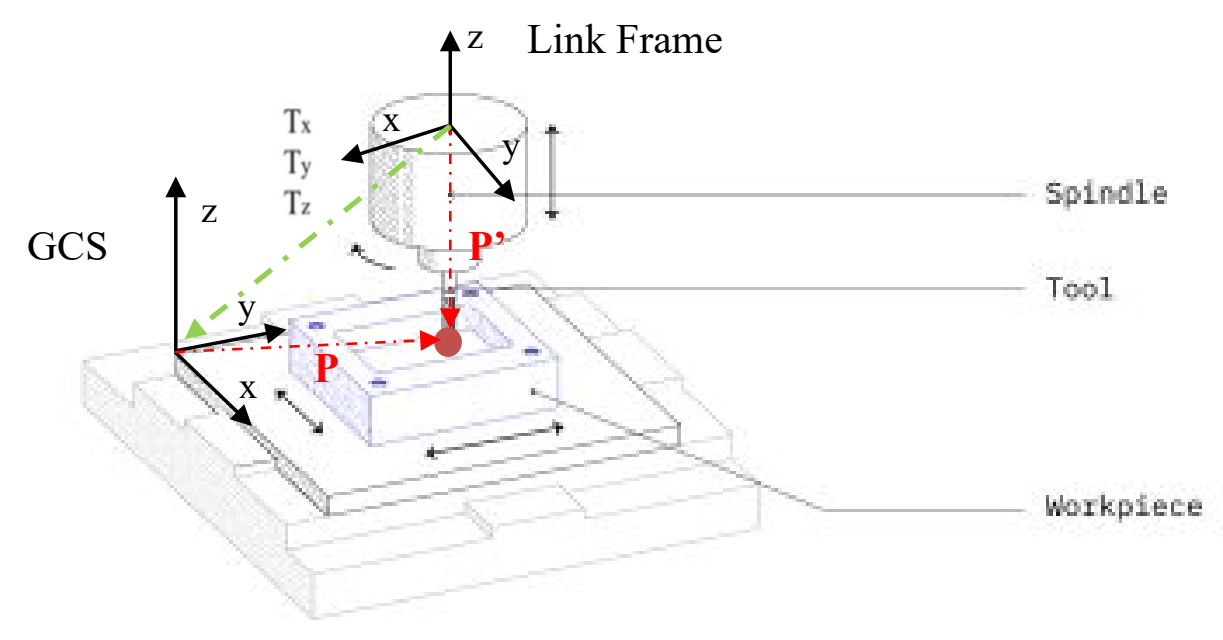

Fig. 6. An example of converting GCS to Link Frame in the context of a machine tool. 


$$
\text { Mapping Matrix }=\left[\begin{array}{cccc}
0 & -1 & 0 & T x \\
1 & 0 & 0 & T y \\
0 & 0 & 1 & T z \\
0 & 0 & 0 & 1
\end{array}\right]
$$

Fig. 7. Mapping matrix for the example.

Another example shows complex rotations of the Link Frame in a rudimentary 5-axis CNC machine. Fig. 8 (a) depicts the Link Frame of the spindle initially in an upright position that is aligned with the GCS. After undergoing a series of two rotations, it reaches the orientation state as depicted in Fig. 8 (b).

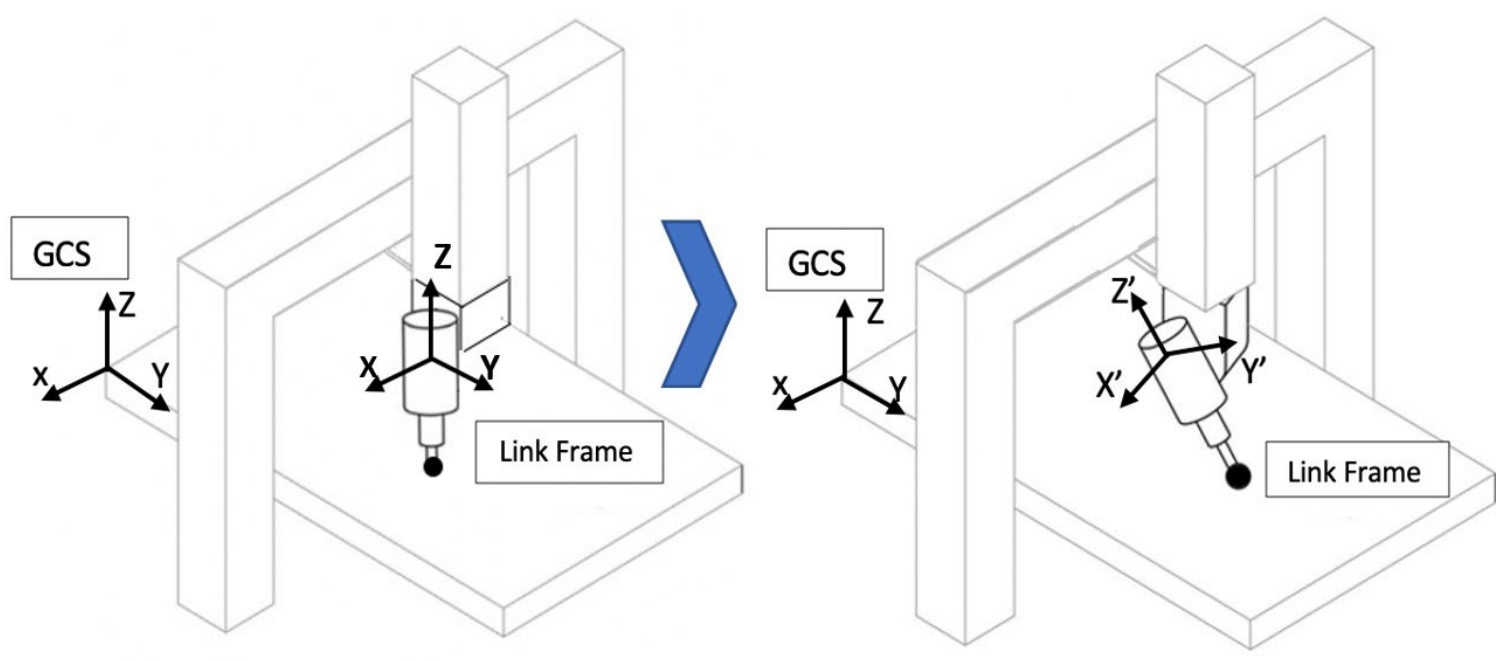

(a)

(b)

Fig. 8. An Example of multiple rotations.

As shown in Fig. 9, the two rotations include $\alpha$ about $\mathrm{X}$-axis and $\beta$ about Z-axis. $\alpha$ and $\beta$ can be any angles, but in this example, we assume that $\alpha$ is $90^{\circ}$ and $\beta$ is $45^{\circ}$. The resulting rotation matrix is solved using trigonometry as shown in Fig. 10. 


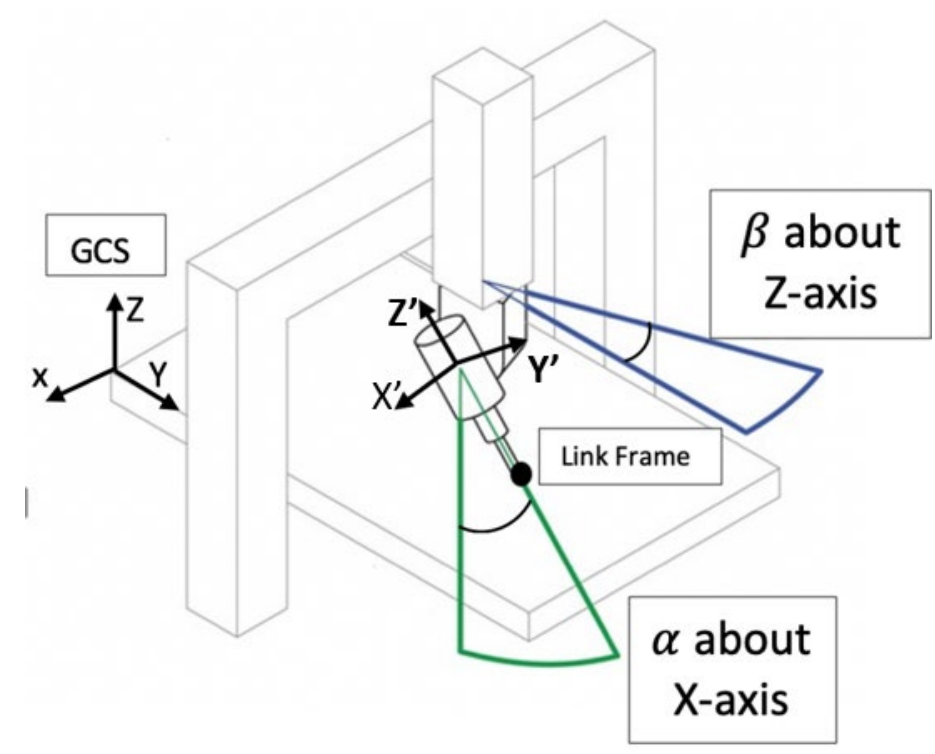

Fig. 9. Rotation angles to reach Fig. 8 (b) state [22].

$$
\text { Mapping Matrix }=\left[\begin{array}{cccc}
\sin (45) & \cos (45) & 0 & T_{x} \\
0 & 0 & 1 & T_{y} \\
\cos (45) & -\sin (45) & 0 & T_{z} \\
0 & 0 & 0 & 1
\end{array}\right]
$$

Fig. 10. Resulting mapping matrix of the rotation example.

The coordinate system differential variation matrix can describe the influence of differential motion of a coordinate system on other coordinate systems. Also, uncertainties and errors introduced by non-uniform thermal expansion of the machine structure and static/dynamic load can also cause a shift (translation or rotation) in the working coordinate system and create the potential for compounding geometric errors and high error margins in the machining process. The same mapping approach can be used to adjust the shift and correct the deviation. For example, by retrieving prior machining data such as measurements of deflection based on various cutting forces, working coordinate systems can be adjusted using the mapping in the model for the given anticipated shift in the coordinate system [23].

\section{Digital Twins of Machine Tools}

As discussed in Section 3, there are multiple uncertainties and potential errors during model development, execution, and maintenance processes. We focus on the translation 
errors and discuss the mapping mechanism for correctly converting coordinate systems in Section 4. This mapping mechanism is a fundamental and critical method to help ensure the machine model accuracy and the machining process effectiveness. For example, as one of the most important factors that affect the machining accuracy and the precision of parts. Geometric errors can be compensated by using homogeneous transformation and differential change between coordinate systems. Applying this method in digital twin modeling will enable constant monitoring, detecting, and correcting for this type of errors. Indeed, digital twins of machine tools will play an important role for dynamically addressing most of the uncertainty issues discussed in Section 3.

Digital twins of machine tools are usually developed using CAx tools and real-time operational data such as spindle current and vibration. The operational data are analyzed in the time and frequency domains [24]. These data include spindle power, feed axis current, tracking errors, and material removal rate. Dynamic data can be constantly collected through internal control feedback while the machine is running. Physical and geometrical data such as cutting force, temperature, vibration, spatial (volumetric) error, thermal deformation, and surface roughness of the workpiece can be collected by using smart sensors and metrology technologies. Some other parameters may be indirectly calculated using the collected data. Digital twins will use all these real-time data (i.e., the internal control feedback data, external collected data, and calculated parameters) to accurately and dynamically track machine working status, optimize the quality of the part produced, and improve the machining process efficiency.

In addition, real-time input data to the digital twin enables:

- Fine-tuning and updating the digital twin model to improve credibility

- Real-time simulation and virtual commissioning that help design, develop, and validate the controller even without the physical system

- Monitoring the machine health by detecting anomalous performance

- Optimizing the physical machine tool's performance.

Digital twins of machines and machining processes help predict machine tool energy consumption, cutting forces, torque, power, stability, and vibrations. However, there is not a single system that can provide all the solutions. For example, existing tool path simulation tools normally do not include capabilities of addressing surface roughness, tool position dynamics, and spindle dynamics [25]. Therefore, the digital twin may need to include multiple models and tools.

\section{Conclusion and Future Work}

When creating machine tool models, assumptions and simplifications are made. These in turn introduce uncertainties and errors when these machine models are used for decision making. For example, errors in the machine geometry can be caused by thermal deformation, static and dynamic loading, or the wear on components. In addition, there are various CAx tools available to model machine tools, but it is cost-prohibitive for a company to have all these tools and maintain proficiency using them. Converting machine models to a standard representation such as STEP can facilitate their reuse and interoperability. However, during the conversion process, errors may also be introduced. 
This paper identifies the uncertainties and potential errors introduced during machine tool model development and execution, addresses translating errors by introducing coordinate system conversion methodology, provides examples for explaining the methodology, and also discusses digital twins as a better solution to address most of the uncertainties. Digital twins of machine tools and machining processes will allow continuous manufacturing at a faster rate with a lower level of uncertainty.

Future work includes using a real-world problem to demonstrate the model conversion with correct coordinate systems, e.g., converting a machine tool model developed using Creo to STEP format; and implementing machine tool digital twins to address more dynamic issues such as these uncertainties discussed in this paper.

\section{References}

[1] NIST (2020) Product definitions for smart manufacturing. https://www.nist.gov/programs-projects/product-definitions-smart-manufacturing

[2] Mori M., Hansel A., and Fujishima M (2014) Machine tool. The International Academy for Production Engineering, Laperrière L., Reinhart G. (eds) CIRP Encyclopedia of Production Engineering. Springer, Berlin, Heidelberg. https://doi.org/10.1007/978-3-642-20617-7_6533

[3] Li Y (2011) Implementation and evaluation of kinematics mechanism modeling based on ISO 10303 STEP. Master Thesis. Royal Institute of Technology.

[4] Vichare P, Zhang X, Dhokia V, Cheung WM, Xiao W, and Zheng L (2018) Computer numerical control machine tool information reusability within virtual machining systems. The Institution of Mechanical Engineers, Part B: Journal of Engineering Manufacture. 2018;232(4):593-604.

[5] Vichare P, Nassehi A, and Newman S (2009) A unified manufacturing resource model for representation of computerized numerically controlled machine tools. The Institution of Mechanical Engineers, Part B: Journal of Engineering Manufacture. 2009;223(5):463-83.

[6]Zivanovic S, Slavkovic N, Kokotovic B, and Milutinovic D (2007) Machining simulations of virtual reconfigurable 5-axis machine tool. Annals of the Faculty of Engineering Hunedoara. 2017;15(2):189-94.

[7] Bärring, M., Shao, G., Helu, M., and Johansson, B. (2020). A case study for modeling machine tool systems using standard representations. The ITU Kaleidoscope 2020 Industry-Driven Digital Transformation.

[8] Kjellberg, T., Von Euler-Chelpin, A., Hedlind, M, Lundgren, M., Sivard, G., and Chen, D (2009) The machine tool model - a core part of the digital factory. CIRP Annals - Manufacturing Technology. 2009, 58: 425 - 428.

[9] Kramer, T. and Xu, X (2009) STEP in a nutshell. Advanced Design and Manufacturing based on STEP. London: Springer; 2009. p. 1-22.

[10] ISO (2014) ISO 10303-105, Industrial automation systems and integration - product data representation and exchange - part 105: integrated application resource:

kinematics. https://www.iso.org/standard/64294.html 
[11] Li Y, Hedlind M, Kjellberg T, and Sivard G (2015) System integration for kinematic data exchange. International Journal of Computer Integrated Manufacturing. 2015; 28(1): 87-97

[12] ISO (1998) ISO 10303-22:Industrial automation systems and integration-product data representation and exchange - part 22: implementation methods: standard data access interface specification.

[13] Slocum A.H (1992) Precision machine design. Englewood Cliffs. Prentice Hall, N.J.

[14] Longstaff, AP, Fletcher, S., Parkinson, S., and Myers, A (2014) The role of measurement and modelling of machine tools in improving product quality. International Journal of Metrology and Quality Engineering. 4, 177-184 (2014) c EDP Sciences.

[15] Longstaff, AP, Fletcher, S., Poxton, A., and Myers, A (2009) Comparison of volumetric analysis methods for machine tools with rotary axes. LAMDAMAP 2009 (Euspen Ltd), pp. 87- 96.

[16] Wang, SM, Yu, HJ, and Liao, HW (2006) A new high-efficiency error compensation system for CNC multi-axis machine tools. International Journal of Advanced Manufacturing Technology. 28, 518 - 526.

[17] Samir, M. and Tunde, O (2010) A review of machine tool accuracy enhancement through error compensation in serial and parallel kinematic machines. International Journal of Precision Technology 1(3), 251-286.

[18] Laspas, T (2014) Modeling and measurement of geometric error of machine tools: methodology and implementation. Master Thesis. Royal Institute of Technology.

[19]LaCourse, D (2020) CAM coordinate systems explored. Available at https://mecsoft.com/blog/cam-coordinate-systems-explored-2/

[20] Miller, J (2004) Dimensional measurement and mathematical error modeling for machine tools. Available at https://webpages.uncc.edu/ jamiller/machmet/machmet.html

[21] Zeid, I (1991) CAD/CAM Theory and Practice. McGraw-Hill Series in Mechanical Engineering Series.

[22] Robeller, C. and Weinand, Y (2016) A 3D cutting method for integral 1DOF multiple-tab-and-slot joints for timber plates, using 5-axis CNC cutting technology. World Conference of Timber Engineering WCTE 2016. Vienna, Austria.

[23] Liang, JC, Li, HF, Yuan, JX, and Ni, J (1997) A comprehensive error compensation system for correcting geometric, thermal, and cutting force-induced errors. International Journal of Advanced Manufacturing Technology, vol. 13, no. 10, pp. $708-712$.

[24] Armendia, M, Alzaga, A., Peysson, F., Fuertjes, T., Cugnon, F., Ozturk, E., and Flum, D (2019) Machine tool: from the digital twin to the cyber-physical systems. Twin-Control.pp 3-21.

[25] Altintas, Y., Kersting, P., Biermann, D., Budak, E., Denkana, B., and Lazoglu, I 2014) Virtual process systems for part machining operations. CIRP Annals. 63(2), $585-605$. 